\title{
Looking versus seeing: Strategies alter eye movements during visual search
}

\author{
Marcus R. Watson, Allison A. Brennan, Alan Kingstone, and James T. Enns \\ University of British Columbia, Vancouver, British Columbia, Canada
}

\begin{abstract}
Visual search can be made more efficient by adopting a passive cognitive strategy (i.e., letting the target "pop" into mind) rather than by trying to actively guide attention. In the present study, we examined how this strategic benefit is linked to eye movements. Results show that participants using a passive strategy wait longer before beginning to move their eyes and make fewer saccades than do active participants. Moreover, the passive advantage stems from more efficient use of the information in a fixation, rather than from a wider attentional window. Individual difference analyses indicate that strategies also change the way eye movements are related to search success, with a rapid saccade rate predicting success among active participants, and fewer and larger amplitude saccades predicting success among passive participants. A change in mindset, therefore, alters how oculomotor behaviors are harnessed in the service of visual search.
\end{abstract}

Visual search involves the coordination of looking (moving one's gaze to new locations) and seeing (distinguishing targets and nontargets). These two aspects of visual search are distinct from one another because high-acuity vision is possible only in a small region at the center of gaze (the fovea), and only when the eyes are stationary (a fixation). To sample detailed information from an extended scene, the eyes must move abruptly (saccade) from one location to another. In the typical inspection of a scene, this fixation-saccade cycle is repeated 3-4 times/sec.

The efficiency of visual search - how rapidly and accurately the target is found - is typically measured by the time that elapses between the first glimpse of a scene and a response indicating target detection. This entails a direct trading relation between seeing and looking: Longer fixations increase information fidelity from each location at the cost of exploring fewer locations, whereas quickly exploring many locations results in reduced fidelity at each one. Studies comparing human oculomotor behavior with an ideal psychophysical observer have indicated that many participants come close to optimizing this trade-off in search (Najemnik \& Geisler, 2005, 2009).

In the present study, we explored the consequences of adopting particular cognitive strategies on this trading relationship. Several studies have shown that participants who are instructed to search passively search more efficiently than those who are instructed to search actively (complete instructions are in the Method section) (Smilek, Dixon, \& Merikle, 2006; Smilek, Enns, Eastwood, \& Merikle, 2006). Smilek, Enns, et al. (2006) hypothesized that the passive strategy gives automatic processes more influence over spatial attention, whereas the active strategy encourages greater reliance on unnecessary executive processes (cf. Wolfe, Alvarez, \& Horowitz, 2000). This interpretation was bolstered by a second experiment in Smilek, Enns, et al. (2006) showing that search was improved when participants performed a simultaneous task that occupied executive processes.

In the present study, we asked three broad questions concerning cognitive strategies and eye movements. First, is there any relationship between the two at all? It may be that strategy has no effect on eye movements, in that all of the participants use their eyes to sample information in essentially the same way. If so, the passive advantage found by Smilek, Dixon, and Merikle (2006) and Smilek, Enns, et al. (2006) may be purely cognitive, reflecting differences in the way scene information is processed after the eyes have sampled it.

Second, if strategies alter eye movements, which oculomotor measures are affected? We hypothesized that passively instructed searchers will shift their emphasis to looking less and seeing more, spending more time on individual fixations than do active searchers. We also expected differences in other oculomotor behaviors. There are at least two ways one could see more: by expanding the attentional window of each fixation (increasing the useful field of view), or by processing the acquired information more deeply. If passive participants have an increased attentional window, it should permit them to make larger saccades, perhaps even targeting these saccades to land between rather than directly on display items. On the other hand, passive participants may represent information from each fixation more richly, allowing them to categorize newly encountered items as a target or as a distractor more rapidly.

The existing literature on oculomotor behavior and search provides hints but no clear answers to these first

M. R.Watson, marcusw@psych.ubc.ca 
two questions. Consistent with the hypothesis that passive searchers make fewer eye movements, efficient search is often correlated with lower saccade frequency (see, e.g., Becic, Boot, \& Kramer, 2008; Boot, Becic, \& Kramer, 2009; Schoonard, Gould, \& Miller, 1973; Shapiro \& Raymond, 1989; Togami, 1984), and preventing searchers from making any eye movements can sometimes improve search (Klein \& Farrell, 1989; Zelinsky \& Sheinberg, 1997). However, recent reports have indicated that more frequent eye movements can also be advantageous, both when peripheral targets are difficult to distinguish from distractors (Boot et al., 2009), and when searching for items in a natural environment (Brennan, Watson, Kingstone, \& Enns, 2009). The possibility of a wider attentional window is supported by positive correlations between search efficiency and saccade amplitude for some tasks (Jacobs, 1986; Phillips \& Edelman, 2008a, 2008b). However, none of these studies gave participants explicit instructions, as in Smilek, Enns, et al. (2006), so it is not clear what generalizations will apply.

The third broad question we asked was whether, in addition to differences in oculomotor behavior at the group level, individual differences in oculomotor measures (i.e., within strategy groups) will be consistent with a shift in emphasis from looking to seeing. Previous reports have pointed to a wide range of individual variation in saccade rates during search, with considerable stability across different tasks (Boot et al., 2009). Thus, the strategic instructions of Smilek, Dixon, and Merikle (2006) and Smilek, Enns, et al. (2006) may differentially benefit individual styles of eye movements. If so, we expect to find different relationships between oculomotor parameters and search success within each instructional group.

In the present study, we used an eyetracker to monitor the gaze of participants who were instructed to search using either an active or a passive strategy. The search displays were similar to those in Smilek, Enns, et al. (2006), with two modifications. First, instead of presenting items at a constant distance from the center of the display, items were distributed over three different distances in order to test whether strategy effects were influenced by target eccentricity and to encourage a wider range of saccade amplitudes. Second, in one trial block, display items were all one size (standard displays), becoming less discriminable with eccentricity as in Smilek, Enns, et al. (2006); in another block, item size increased linearly with distance from the center (cortical magnification) in order to roughly equate discriminability for all items.

\section{METHOD}

\section{Participants}

Forty-four undergraduate students reporting normal or correctedto-normal vision participated in a 1-h session for course credit. Participants were randomly assigned to each strategy group.

\section{Displays}

Examples of search displays are shown in Figure 1. Displays consisted of a target (a circle with a gap on the left or the right) and two, five, or eight distractors (circles with two gaps). One third of all items were placed on each of three invisible rings, with radii of $1.75,3.5$, and 7 degrees of visual angle. Items were randomly placed on each

\section{A}

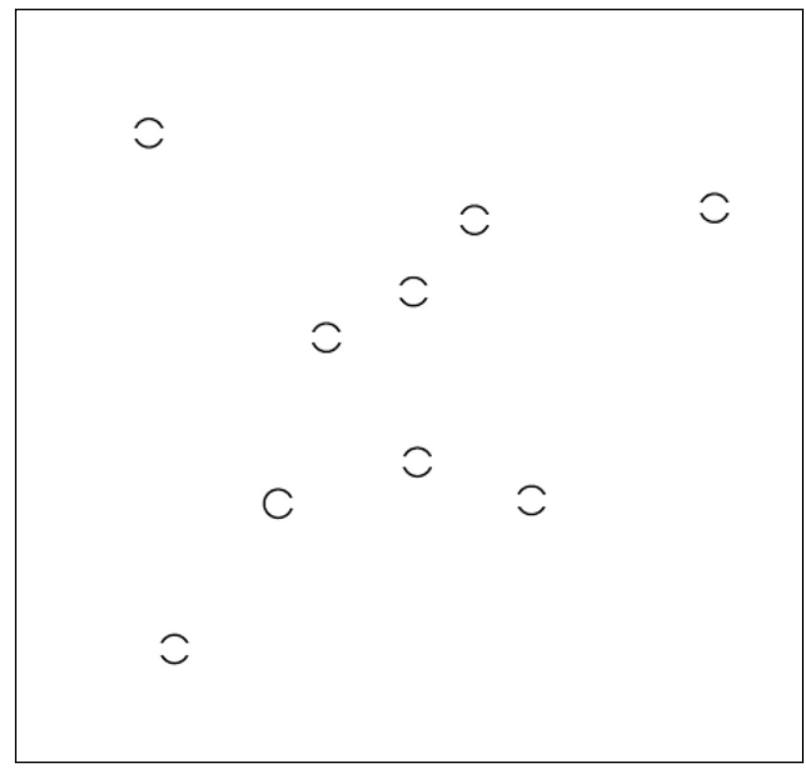

B

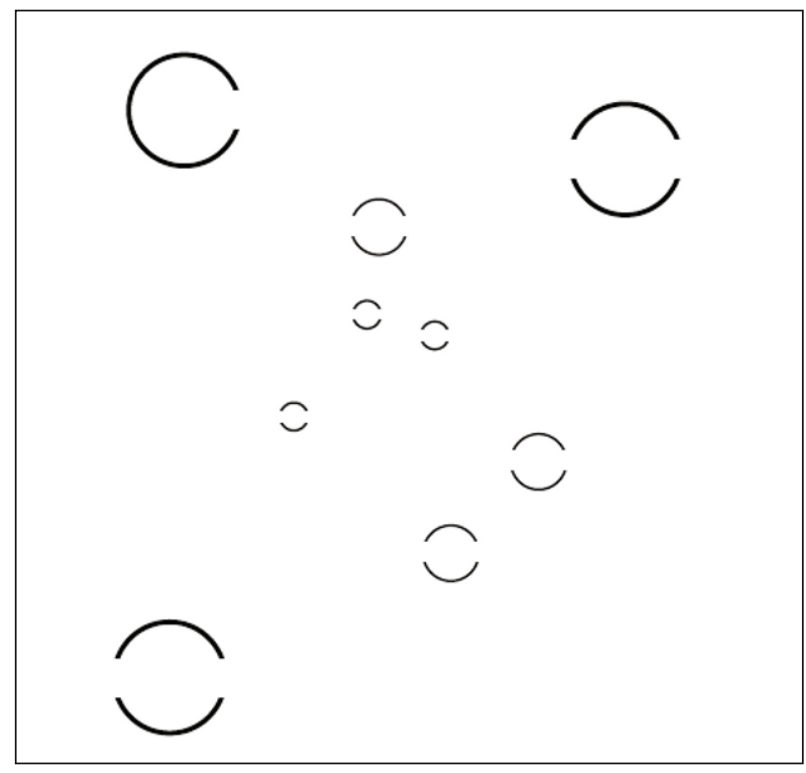

Figure 1. Illustration of the two types of visual search displays seen by participants. (A) Standard displays. (B) Cortically magnified displays. The target shown in each display has a gap on the right.

ring so that they could be no closer than 15 degrees of arc to each other. In one trial block (standard displays), all of the items were the same size $\left(0.5^{\circ}\right.$ with gaps of $\left.0.18^{\circ}\right)$, whereas in another block (magnified displays), item and gap size increased linearly with eccentricity $\left(0.5^{\circ}, 1^{\circ}\right.$, and $2^{\circ}$, with gaps of $0.18^{\circ}, 0.36^{\circ}$, and $0.72^{\circ}$, respectively).

\section{Procedure}

Participants sat at a desk in an unlit room with their heads in a chinrest located $65 \mathrm{~cm}$ from the screen. An SR Research Eyelink 
1000 tracked eye position at $500 \mathrm{~Hz}$. Each session began with a standard nine-point eyetracker calibration. Participants read the task instructions before practicing on 10 standard displays that were repeated if accuracy was less than $90 \%$. Participants were then given strategy instructions, both verbally by the experimenter and as written instructions on screen. The instructions (taken directly from Smilek, Enns, et al., 2006) read as follows for the passive group:

The best strategy for this task, and the one that we want you to use in this study, is to be as receptive as possible and let the unique item "pop" into your mind as you look at the screen. The idea is to let the display and your intuition determine your response. Sometimes people find it difficult or strange to tune into their "gut feelings" but we would like you to try your best. Try to respond as quickly and accurately as you can while using this strategy. Remember, it is very critical for this experiment that you let the unique item just "pop" into your mind. (p. 548)

The instructions for the active group read as follows:

The best strategy for this task, and the one that we want you to use in this study, is to be as active as possible and to "search" for the item as you look at the screen. The idea is to deliberately direct your attention to determine your response. Sometimes people find it difficult or strange to "direct their attention" but we would like you to try your best. Try to respond as quickly and accurately as you can while using this strategy. Remember, it is very critical for this experiment that you actively search for the unique item. (p. 549)

The participants' task was to find the target (present on all trials) and to press a key to indicate whether it had a gap on the left or the right side. Testing consisted of two blocks of 144 trials (one for each display type), with the order counterbalanced across participants and eyetracker calibration between blocks. Within each block, the display size (three, six, or nine items), target eccentricity (inner, middle, or outer ring), and target gap (left, right) were counterbalanced. Each trial began with a blank screen for $100-750 \mathrm{msec}$, followed by the display, which remained on view until a key was pressed or $4,500 \mathrm{msec}$ had elapsed. Feedback was presented at the center of the screen for $200 \mathrm{msec}$ ("+" for correct and "-" for incorrect or failures to respond) following each response.

\section{RESULTS}

\section{Strategy Instructions Affect Search Performance}

Figure 2A shows the mean correct response time (RT) and mean proportion errors (PE), as a function of display size, for participants in the two strategy groups. Strategy instructions had an effect similar to that reported in Smilek, Enns, et al. (2006): In comparison with active instructions, passive instructions were associated with more rapid RT overall, shallower increases in RT with display size, and a tendency toward more errors. These observations were supported by ANOVAs for both RT and PE, in which strategy (active, passive) was a between-participants factor, whereas display type (standard, magnified), display size (three, six, nine), and target eccentricity (one, two, three) were within-participants factors. The main effect of strategy was significant for RT $[F(1,42)=6.80, p<.05]$, as was the strategy $\times$ display interaction $[F(2,84)=4.29$, $p<.05]$. For PE only, the main effect of strategy was significant $[F(1,42)=5.97, p<.05]$.

Because passive instructions were associated with both lower RT and higher PE, one must be cautious in interpreting this as an overall advantage. Perhaps instructions
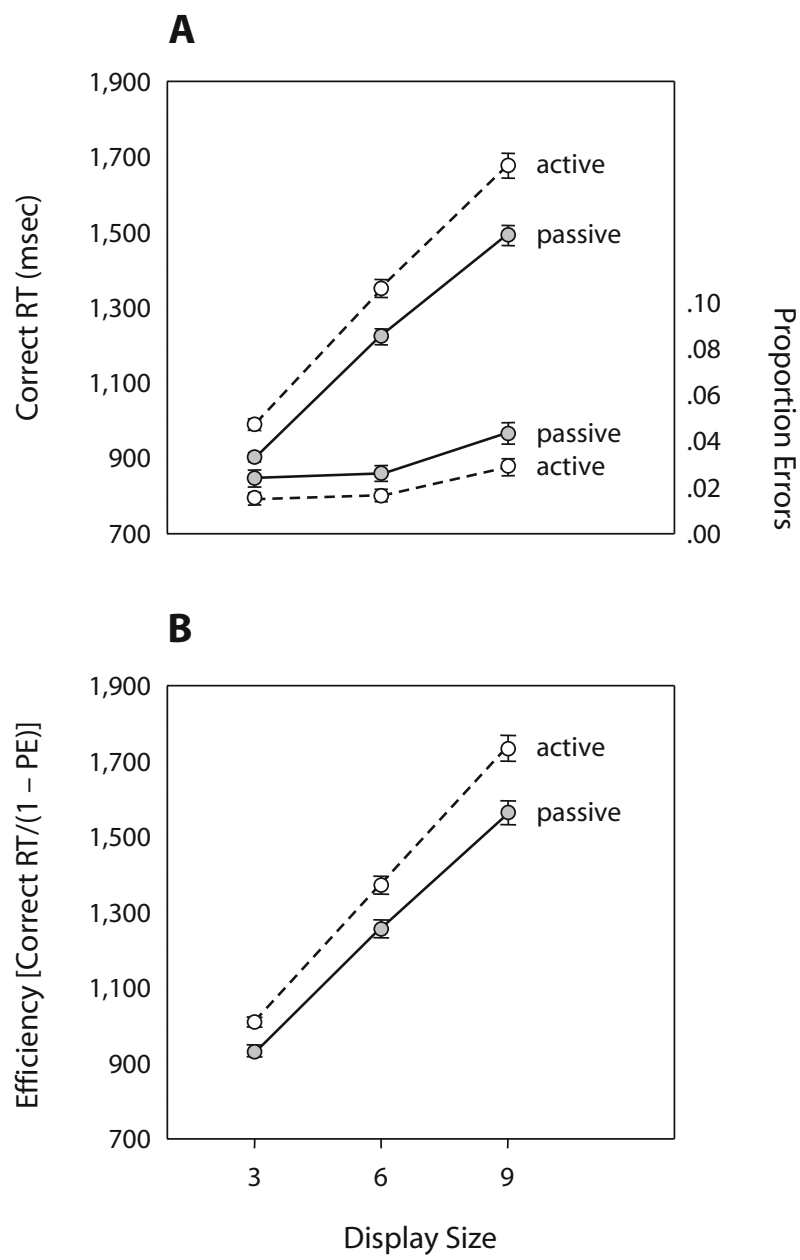

Figure 2. (A) Correct response time (RT) and proportion errors (PE) in the search task. (B) A combined measure of search efficiency: RT divided by $(1-P E)$.

simply moved participants in different directions along a speed-accuracy trading function. One way to control for this is to combine RT and PE in a single measure of search efficiency, by dividing the mean correct RT by the mean proportion correct for each participant in each condition (Smilek, Enns, et al., 2006; Townsend \& Ashby, 1983). Doing this corrects RT for accuracy differences in an intuitive way: When accuracy is perfect, efficiency will be identical to mean RT, and as accuracy decreases, the combined score increases. Thus, efficiency scores are like RT in that smaller values indicate better performance. The main assumption underlying the use of these scores is that RT and accuracy are related linearly, which was supported in the present data by a correlation between RT and PE in the 36 conditions of the ANOVA $[r(34)=-.338$, $p<.05]$.

The efficiency scores still show a clear advantage for passive over active instructions, indicating that the benefit of passive instructions went beyond moving participants along a speed-accuracy function (see Figure 2B). An ANOVA on the combined scores involving the same aforementioned factors revealed a significant main effect 
of strategy $[F(1,42)=5.26, p<.05]$, a marginal interaction of strategy $\times$ display size $[F(1,42)=2.80, p<$ $.07]$, and no other interactions involving strategy. Each of the stimulus factors had expected effects (e.g., the efficiency score decreased with display size and with target eccentricity on standard displays, but the target eccentricity effect was reduced in cortically magnified displays), but none of them interacted significantly with strategy instructions (all $p \mathrm{~s}>.10$ ). The search efficiency scores and oculomotor measures were therefore averaged over these factors in all subsequent analyses.

\section{Strategy Instructions Affect Oculomotor Behavior of the Group}

Figure 3 shows the typical number of saccades made by participants, both before fixating the target the first time and after fixating it. The target location was defined as a circular region centered on the target item and extending $0.25^{\circ}$ beyond the contours of the item. Passively instructed participants were more likely than actively instructed participants to fixate the target in three or fewer saccades, whereas this pattern reversed when four or more saccades were made $\left[\chi^{2}(10)=65.44, p<.001\right]$ (see Figure 3A). Actively instructed participants continued to make more saccades even after the target had been fixated $\left[\chi^{2}(8)=\right.$ 291.67, $p<.001$ ] (Figure 3B).

More detailed analyses examined oculomotor measures by dividing each trial into three epochs, as is shown in Figure 4 . The first epoch refers to cognitive events that occur before any eye movements are made and is indexed by first saccade latency (in milliseconds), the time from display onset until the first saccade (see Figure 4B). Isolating this period is important, since apparent differences in fixation duration during search are sometimes based entirely on first saccade latency (Zelinsky \& Sheinberg, 1997). This measure shows that participants in the pas- sive group gazed longer at the center of the display before making a first saccade than did those in the active group $[t(42)=2.65, p<.05]$, consistent with a greater emphasis on seeing.

Five measures indexed the second epoch: Fixation duration (in milliseconds) was the time the eyes remained still between saccades; saccade rate was the number of eye movements made to new locations per second; saccade amplitude (in degrees) was the size of the eye movement; saccade duration (in milliseconds) was the duration of the eye movement; and target saccade amplitude (in degrees) was the size of the first saccade made to the target location. There were no simple group differences within the five oculomotor measures taken in the second trial epoch (all $p \mathrm{~s}>.13$ ), although there were group-based influences on the relations between these oculomotor measures and search efficiency, as will be described in the next section.

The third epoch in Figure 4 (lower panel) was indexed by target saccade to response (in milliseconds), the time between the first saccade to the target and the manual response to the target (a buttonpress). Here, participants in the passive group were faster to respond, once they had fixated the target, than those in the active group $[t(42)=$ $2.95, p<.01]$, showing that they were better prepared to see the target once they had acquired it with the appropriate saccade.

To test whether passive participants had a wider useful field of view, we compared the groups for the average distance across all fixations to the center of the screen (reasoning that a larger field of view would encourage searchers to fixate at the center, in order to see more items at once), and in the average distance of all fixations to the nearest item on the screen (reasoning that a larger field of view would allow searchers to target fixations between items, rather than directly on them). Neither analysis revealed significant effects of strategy (both $p \mathrm{~s}>.50$ ).
A

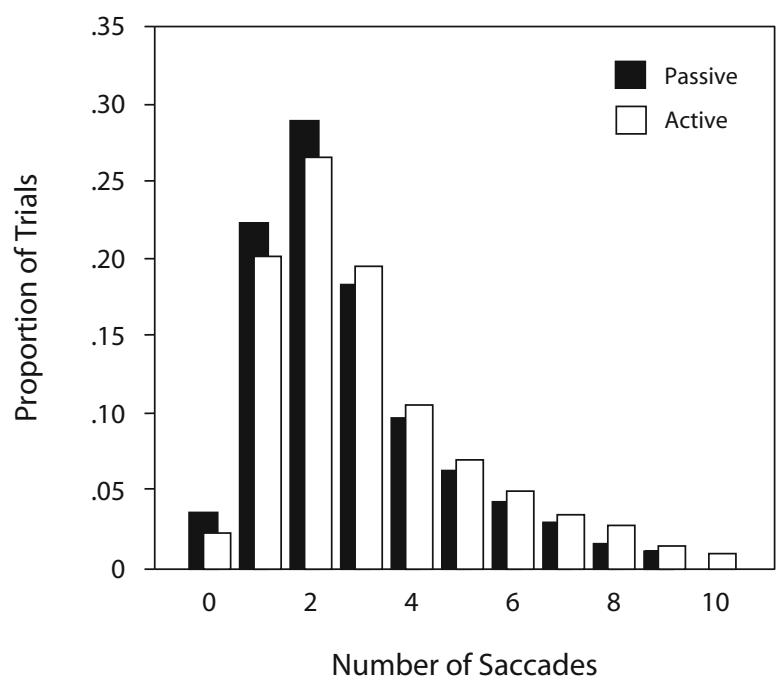

B After Target Fixation

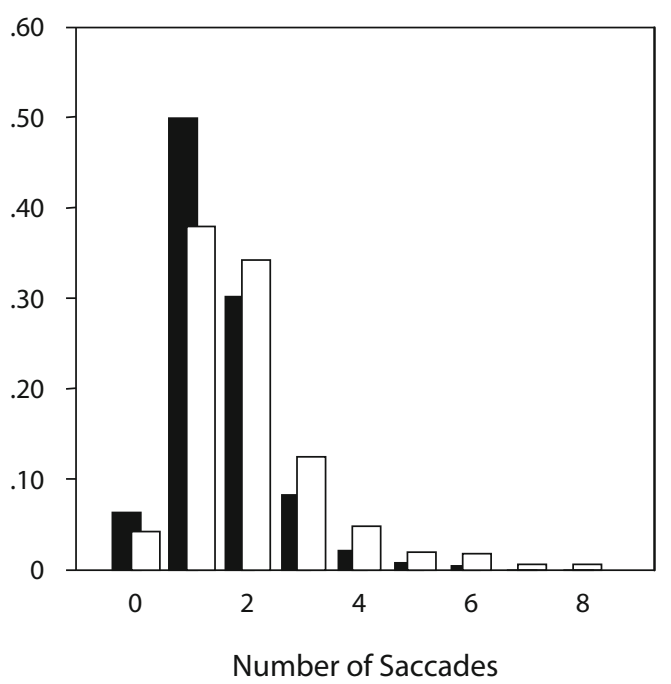

Figure 3. Relative frequency of saccades made by participants (A) before first fixating the target and (B) after fixating the target. 
A

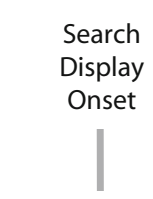

First Saccade

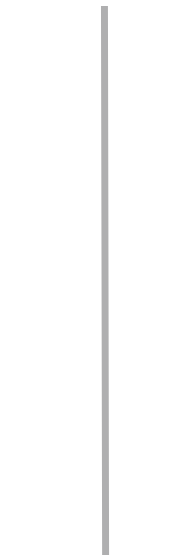

Saccades

to Target

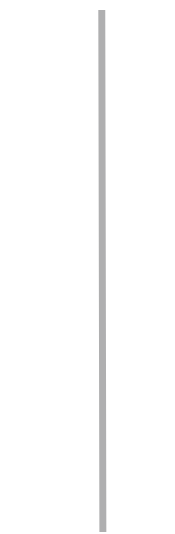

Target Saccade to Response

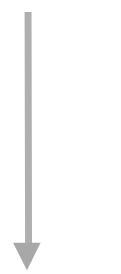

B

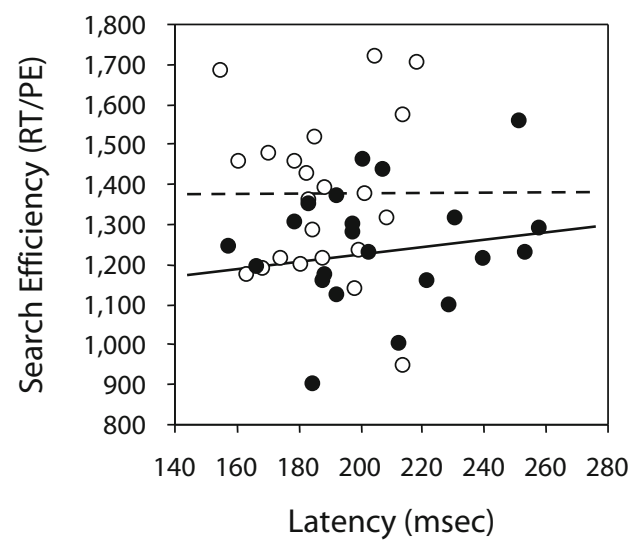

C

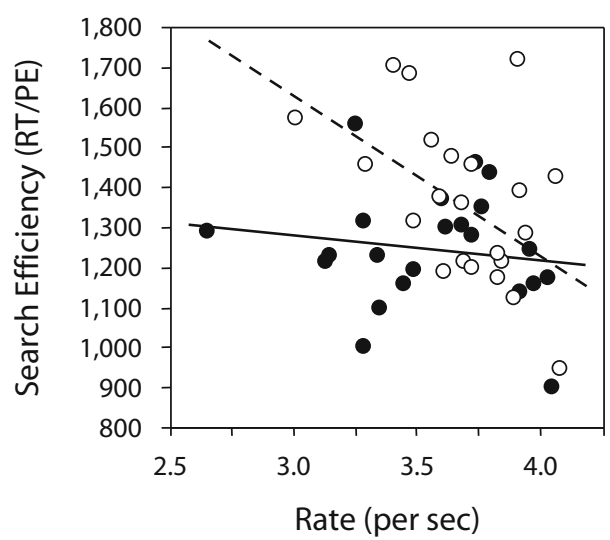

D

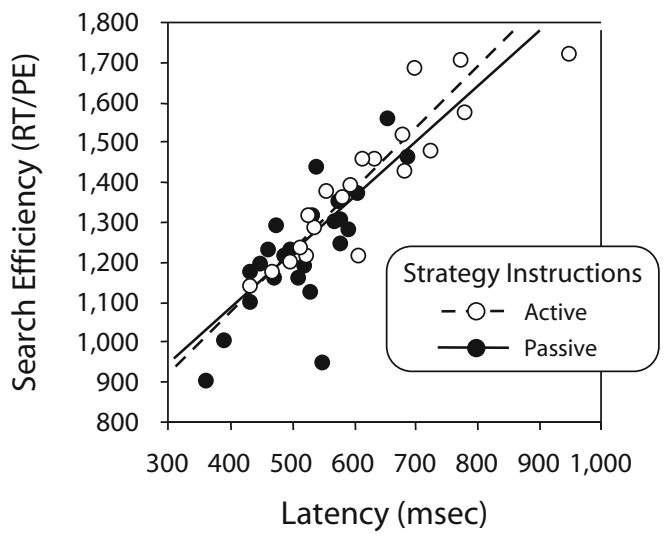

Figure 4. (A) Each trial was divided into three epochs of oculomotor measures. The figure shows scattergrams of search efficiency (the combined score from Figure 2B) as predicted by three oculomotor measures: (B) latency of first saccade, (C) saccade rate, and (D) target saccade to response. Regression lines are shown separately for the 22 participants in each of the active and passive strategy groups. RT, response time; PE, proportion errors.

We also tested whether the oculomotor differences we found were associated with strategy over and above differences in search efficiency. To eliminate group differences in efficiency, we removed 4 passive participants with the low- est mean efficiency scores, and 4 active participants with the highest scores, leading to mean scores of 1,298 (passive) and 1,304 (active) $(p>$.75). The pattern of results was unchanged with two exceptions: The remaining passive 
searchers had a slower saccade rate [passive mean $=3.53$ $\mathrm{sacc} / \mathrm{sec}$; active mean $=3.73 \mathrm{sacc} / \mathrm{sec} ; t(34)=2.19, p<$ $.05]$ and no longer had a faster target saccade to response time $(p>.10)$ than the remaining active searchers.

\section{Strategy Instructions Affect Individual Differences in Oculomotor Behavior}

Our final analyses explored the relations between oculomotor measures and search efficiency for participants within each strategy group. The main findings were as follows: (1) Most of the individual differences in search efficiency could be accounted for by oculomotor measures, and (2) the oculomotor measures that best predicted search efficiency were consistent with a seeing bias for passively instructed participants and a looking bias for actively instructed participants.

As is shown in Table 1, a multiple regression using all seven measures to predict search efficiency accounted for $89 \%$ of the variance of the passive group and $82 \%$ of the active group. The single most important measure in both groups was target saccade to response. A model using this single measure accounted for $74 \%$ (passive) and $72 \%$ (active) of the between-participants variation in search efficiency (both $p \mathrm{~s}<.001$ ). The two groups differed, however, in which other measures were important. We constructed reduced models for both groups by selecting the variables that made the largest individual contribution to the full model, adding variables until the resulting model was not statistically different from the complete model. For the passive group, adding target saccade amplitude increased the variance explained to $83 \%$, whereas for the active group, adding saccade rate increased the variance explained to $79 \%$. In both cases, the single extra variable made the model statistically indistinguishable from the complete model. This indicates that the most successful searchers in the passive group also made the largest amplitude saccades to the target, whereas those in the active group made saccades at the most rapid rate, consistent with the passive group's placing more emphasis on seeing and the active group's placing more emphasis on looking.

In support of the generality of these findings, we observed the same pattern of correlations when, instead of using the mean efficiency score of each participant as the dependent variable, we used the mean slope of the efficiency score (i.e., the change in efficiency that occurs as a function of display size).

\section{DISCUSSION}

The present study documented a direct link between strategy instructions for visual search and the eye movements made during search. Although correlations have previously been reported for oculomotor measures and search efficiency (see, e.g., Boot et al., 2009), the present study demonstrated that randomly assigning participants to different strategies - with instructions that make no reference to the eyes-leads to systematic differences in oculomotor behavior. Moreover, the present results go further than merely showing that strategies influence looking behavior; they show that the relations between eye movements and search success are altered when participants adopt different strategies.

Table 1

Multiple Regression Models Using Oculomotor Measures to Predict Individual Differences in Search Efficiency in the Two Strategy Groups

\begin{tabular}{|c|c|c|c|c|c|c|}
\hline \multirow[b]{3}{*}{ Oculomotor Predictors } & \multirow{2}{*}{\multicolumn{2}{|c|}{$\frac{\text { Full Model }}{\text { Seven Predictors }}$}} & \multicolumn{4}{|c|}{ Reduced Models } \\
\hline & & & \multicolumn{2}{|c|}{ Two Predictors } & \multicolumn{2}{|c|}{ One Predictor } \\
\hline & Partial $r$ & $p$ & Partial $r$ & $p$ & Simple $r$ & $p$ \\
\hline \multicolumn{7}{|c|}{ Active Strategy } \\
\hline First saccade latency & -.552 & .027 & & & & \\
\hline Fixation duration & .278 & .300 & & & & \\
\hline Saccade rate $(\mathrm{deg} / \mathrm{sec})$ & -.695 & .003 & -.492 & .024 & & \\
\hline Saccade duration & -.640 & .008 & & & & \\
\hline Saccade amplitude & .494 & .052 & & & & \\
\hline Target saccade amplitude & -.369 & .159 & & & & \\
\hline Target to response & .909 & .001 & .846 & .001 & .848 & .001 \\
\hline$R^{2}$ & \multicolumn{2}{|c|}{.892} & \multicolumn{2}{|c|}{.787} & \multicolumn{2}{|c|}{.719} \\
\hline \multicolumn{7}{|c|}{ Passive Strategy } \\
\hline First saccade latency & .202 & .452 & & & & \\
\hline Fixation duration & -.120 & .658 & & & & \\
\hline Saccade rate $(\mathrm{deg} / \mathrm{sec})$ & -.231 & .389 & & & & \\
\hline Saccade duration & -.069 & .798 & & & & \\
\hline Saccade amplitude & .123 & .650 & & & & \\
\hline Target saccade amplitude & -.562 & .023 & -.582 & .024 & & \\
\hline Target to response & .932 & .001 & .910 & .001 & .862 & .001 \\
\hline$R^{2}$ & \multicolumn{2}{|c|}{.892} & \multicolumn{2}{|c|}{.830} & \multicolumn{2}{|c|}{.743} \\
\hline
\end{tabular}

Note-A full model involving seven oculomotor predictors was compared with two reduced models, one based on two predictors that were the largest contributors in the full model, and one based on the single predictor that was the largest contributor. Partial $r$ is a relative measure of variation for each predictor; $p$ refers to the associated significance level; and $R^{2}$ is the proportion of variance in the individual search efficiency scores that is accounted for by the predictors in each model. 
In general, we interpret our results as indicating that passive instructions encourage participants to place an emphasis on seeing (processing the information available in a fixation), whereas active instructions place an emphasis on looking (making saccades to new locations). Furthermore, passive participants' improved performance is the result of more efficiently processing information in a fixation, rather than of accessing information from a wider region. This interpretation was supported by several analyses. Passive searchers do not generally make larger saccades than active searchers, nor do they target their fixations further away from individual items in order to access more information from each fixation, nor do they target fixations more centrally in the display. Instead, they make longer initial fixations, make fewer fixations overall, and are less likely to continue making additional fixations after fixating the target. Furthermore, peripheral target discriminability has no effect on the passive advantage. Active participants, on the other hand, are less able to react once they fixate the target, indicating that they are less prepared than the passive participants to process new items.

Perhaps even more important than these simple group differences is the finding that the oculomotor measures that best predicted search efficiency at an individual level depended on the adopted strategy. For all searchers, responding quickly to the target after first fixating it was critical to search success, but this is where the similarity ended. The most efficient passive searchers were those making the largest amplitude saccades to the target, whereas the most efficient active searchers were those who made saccades to new locations most rapidly. We interpret this to mean that the most efficient passive searchers are those who use the information available from their longer initial fixations to guide subsequent search, permitting fewer and more direct (and hence larger) saccades to the target. The additional information from the first fixation is not available to active searchers, so the option of larger saccades is not available to them.

These results must also be considered in tandem with the observation that there was much overlap in search success between participants in each strategy group. The fact that some participants in each group were able to search equally well, as measured by response speed and accuracy, while nonetheless engaging in a different saccade-fixation trading relation, implies that there is more than one way to guide the eyes during effective search. That is, the results show that both rapid saccades to new locations and calm consideration of the information within a fixation can lead to similar search success with the present displays.

A challenge for the future will be to determine whether the trade-off seen in the present study for emphasis on fixations versus saccades holds under different display types and search environments. Perhaps different saccade-fixation emphases led to similar search efficiency in the present study only because the displays we tested allowed participants to gain considerable informa- tion, even at the more distant item locations, when the eyes were still. Future studies would do well to explore the effect of search instructions under a variety of environmental conditions, as our laboratory has begun doing (Brennan et al., 2009).

\section{AUTHOR NOTE}

This research was supported by a Discovery Research Grant from the Natural Sciences and Engineering Research Council (Canada) to J.T.E. and by an SSHRC Doctoral Award to M.R.W. Address correspondence to M. R. Watson, Department of Psychology, University of British Columbia, Vancouver, BC, V6T1Z4 Canada (e-mail: marcusw@psych.ubc.ca).

\section{REFERENCES}

Becic, E., Boot, W. R., \& Kramer, A. F. (2008). Training older adults to search more effectively: Scanning strategy and visual search in dynamic displays. Psychology \& Aging, 23, 461-466.

Boot, W. R., Becic, E., \& Kramer, A. F. (2009). Stable individual differences in search strategy? The effect of task demands and motivational factors on scanning strategy in visual search. Journal of Vision, 9(3, Art. 7), 1-16.

Brennan, A. A., Watson, M. R., Kingstone, A., \& Enns, J. T. (2009) From lab to life: The effect of cognitive strategies on real-world search. Journal of Vision, 9(8), 1203a. doi:10.1167/9.8.1203

JACOBS, A. M. (1986). Eye-movement control in visual search: How direct is visual span control? Perception \& Psychophysics, 39, 47-58.

Klein, R., \& Farrell, M. (1989). Search performance without eye movements. Perception \& Psychophysics, 46, 476-482.

NAJemnik, J., \& GeISler, W. S. (2005). Optimal eye movement strategies in visual search. Nature, 434, 387-391.

Najemnik, J., \& Geisler, W. S. (2009). Simple summation rule for optimal fixation selection in visual search. Vision Research, 49, 12861294.

Phillips, M. H., \& Edelman, J. A. (2008a). The dependence of visual scanning performance on saccade, fixation, and perceptual metrics. Vision Research, 48, 926-936.

Phillips, M. H., \& Edelman, J. A. (2008b). The dependence of visual scanning performance on search direction and difficulty. Vision Research, 48, 2184-2192.

Schoonard, J. W., Gould, J. D., \& Miller, L. A. (1973). Studies of visual inspection. Ergonomics, 16, 365-379.

ShapiRo, K. L., \& RaYmond, J. E. (1989). Training of efficient oculomotor strategies enhances skill acquisition. Acta Psychologica, 71, 217-242.

Smilek, D., Dixon, M. J., \& Merikle, P. M. (2006). Revisiting the category effect: The influence of meaning and search strategy on the efficiency of visual search. Brain Research, 1080, 73-90.

Smilek, D., Enns, J. T., Eastwood, J. D., \& Merikle, P. M. (2006). Relax! Cognitive strategy influences visual search. Visual Cognition, 14, 543-564.

Togami, H. (1984). Affects on visual search performance of individual differences in fixation time and number of fixations. Ergonomics, 27, 789-799

Townsend, J. T., \& Ashby, F. G. (1983). Stochastic modeling of elementary psychological processes. New York: Cambridge University Press.

Wolfe, J. M., Alvarez, G. A., \& Horowitz, T. S. (2000). Attention is fast but volition is slow. Nature, 406, 691 .

Zelinsky, G. J., \& SheINBERG, D. L. (1997). Eye movements during parallel-serial visual search. Journal of Experimental Psychology: Human Perception \& Performance, 23, 244-262.

(Manuscript received November 3, 2009; revision accepted for publication February 11, 2010.) 